\title{
FITOTECNIA
}

\section{MODOS DE APLICAÇÃO DE REGULADOR VEGETAL NO ALGODOEIRO, CULTIVAR IAC-22, EM DIFERENTES DENSIDADES POPULACIONAIS E NÍVEIS DE NITROGÊNIO EM COBERTURA ${ }^{(1)}$}

\author{
ENES FURLANI JUNIOR ${ }^{(2)}$; NELSON MACHADO DA SILVA ${ }^{(3,5)}$; LUIZ HENRIQUE CARVALHO ${ }^{(3)}$; \\ NELSON BORTOLETTO ${ }^{(4)}$; JOSÉ CARLOS SABINO(4); ${ }^{(2 E N I Z A R T ~ B O L O N H E Z I ~}{ }^{(4)}$
}

\begin{abstract}
RESUMO
Este trabalho foi desenvolvido nos anos agrícolas de 1996/97, 1997/98, 1998/99 e 1999/00, em experimentos de campo nas localidades de Ribeirão Preto, Tietê, Guaíra, Campinas e Ilha Solteira, no Estado de São Paulo. O delineamento experimental empregado foi o de blocos ao acaso com doze tratamentos arranjados em um esquema fatorial $3 \times 2 \times 2$, visando estudar densidade de plantas $(6 ; 10$ e 14 plantas por metro); doses de nitrogênio em cobertura (40 e $60 \mathrm{~kg} \cdot \mathrm{ha}^{-1}$ de N) e modos de aplicação de regulador vegetal, com aplicação única, no início do florescimento ou parcelada em 4 vezes, desde o desbaste. O sistema de aplicação parcelada propiciou altura média de planta inferior e massa média de capulhos superior àquele obtido com a aplicação única do regulador. A aplicação da maior dose de $\mathrm{N}$ implicou obtenção dos maiores valores de $\mathrm{N}$ foliar e produtividade de algodão em caroço, bem como menor porcentagem de fibra. O aumento da densidade de plantas de 6 para 10 e 14 plantas por metro, ocasionou redução da porcentagem de fibra e da massa de capulhos.
\end{abstract}

Palavras-chave: algodão, Gossypium hirsutum L., cv. IAC 22, regulador de crescimento, nitrogênio, população de plantas.

\section{ABSTRACT \\ TYPES OF GROWTH REGULATOR APPLICATION, PLANTING DENSITIES AND NITROGEN LEVELS FOR THE COTTON CULTIVAR IAC 22.}

The present work was developed during the growing seasons of 1996/97, 1997/98, 1998/99 and 1999/00 at Ribeirão Preto, Tietê, Guaíra, Campinas and Ilha Solteira, State of São Paulo, Brazil. The experimental design was the completely casualized blocks, using the factorial arrangement with twelve treatments $(3 \times 2 \times 2)$ and four replications. The objective of this work was to study plant density $(6 ; 10$ and 14 plants $/ \mathrm{m}$ ); nitrogen levels at broadcasting (40 and $60 \mathrm{~kg}$.ha ${ }^{-1}$ of $\mathrm{N} / \mathrm{ha}$ ) and types of growth regulator application (single application at flowering or splitting in four times, since the thinning). The split application conduced to lower height of plants and greater weight of bolls when compared to the single application of the growth regulator. The application of the highest level of $\mathrm{N}$, increased the leaves nitrogen content in and cotton yield, and reduced the lint percentage. increasing plant density from 6 to 10 and 14 plants/m, reduced lint porcentage and bolls weight.

Key-words: Gossypium hirsutum, growt regulator, nitrogen, plant density.

$\left({ }^{1}\right)$ Recebido para publicação em 24 de junho de 2002 e aceito em 24 de fevereiro de 2003.

( $\left.{ }^{2}\right)$ Faculdade de Engenharia/UNESP/Campus de Ilha Solteira, Caixa Postal 31, 15385-000 Ilha Solteira (SP). E-mail: enes@agr.feis.unesp.br

$\left({ }^{3}\right)$ Centro de Análise e Pesquisa Tecnológica do Agronegócio de Grãos e Fibras, Instituto Agronômico, Campinas (SP).

$\left({ }^{4}\right)$ Departamento de Descentralização de Desenvolvimento, APTA/SAA.

$\left({ }^{5}\right)$ Com bolsa de pesquisa do CNPq. 


\section{INTRODUÇÃO}

A escassez e/ou baixa qualidade da mão-deobra e a crescente utilização de grandes áreas para a produção do algodão têm gerado algumas indagações técnicas podendo-se citar, entre elas, a possibilidade de utilização de maiores densidades de plantas em conseqüência da eliminação do desbaste (CARVAlHo e FURLANI JUNIOR, 1996).

As tentativas nesse caso têm gerado alguns problemas, como excessivo acamamento das plantas (sobretudo nas variedades mais recentes, de maior produtividade), apodrecimento anormal dos frutos e a dependência da carga do ponteiro para boa produtividade.

Um manejo diferenciado da cultura, mediante a adequação da adubação nitrogenada para atender à alta população de plantas, e o uso de regulador vegetal a fim de impedir o excessivo crescimento vegetativo, consistem em aspectos técnicos de relevante importância que devem ser estudados. Ademais, o desenvolvimento crescente da colheita motomecanizada, requer um ajuste geral das técnicas culturais do algodoeiro.

O objetivo deste trabalho foi avaliar os efeitos da adubação nitrogenada e o modo de aplicação de regulador vegetal sobre o comportamento produtivo da cultivar de algodão (Gossypium hirsutum L. ), IAC 22, em diferentes densidades populacionais.

\section{MATERIAL E MÉTODOS}

O presente trabalho foi desenvolvido nos anos agrícolas de 1996/97, 1997/98, 1998/99 e 1999/00, em experimentos de campo, em Ribeirão Preto, Tietê, Guaíra, Campinas e Ilha Solteira. O delineamento experimental empregado foi o de blocos ao acaso com doze tratamentos arranjados em um esquema fatorial $3 \times 2 \times$ 2 , visando estudar na cultivar de algodoeiro IAC 22: densidade de plantas (6; 10 e 14 plantas por metro); doses de nitrogênio em cobertura (40 e $60 \mathrm{~kg}^{\circ} \mathrm{ha}^{-1}$ de N) e modos de aplicação de regulador vegetal, com aplicação única, no início do florescimento ou parcelada em quatro vezes, desde o desbaste.

Cada tratamento constou de quatro repetições em um total de 48 parcelas, área útil de 19,2 a $20 \mathrm{~m}^{2}$ por parcela (4 x $5 \mathrm{~m}$ ), espaçamento entre linhas de semeadura de 0,9 ou $1,0 \mathrm{~m}$, dependendo da localidade e conforme requerido pela colheita mecânica. Utilizou-se a adubação básica de semeadura recomendada para a cultura, aplicando-se a dose indicada pela análise de terra das respectivas localidades, de acordo com as recomendações de SiLva e RaIJ (1996).
Aos 30 dias da emergência das plântulas, efetuou-se o desbaste do excesso de plantas, deixando-se 6, 10 e 14 plantas por metro, em função dos tratamentos. Nessa ocasião, efetuou-se a primeira cobertura com $40 \mathrm{~kg} \cdot \mathrm{ha}^{-1}$ de N. Ao redor de 50 dias, foi efetuada a segunda cobertura $\left(20 \mathrm{~kg} \cdot \mathrm{ha}{ }^{-1}\right.$ de N) nos tratamentos correspondentes .

O desenvolvimento vegetativo do algodoeiro foi controlado pelo emprego de regulador vegetal (cloreto de mepiquat) aplicado de duas formas: dose total (1 L.ha ${ }^{-1}$ do produto comercial), por ocasião do início do florescimento; ou parceladamente, a partir do desbaste, com aplicação a cada 10 dias, em ordem de 0,$1 ; 0,2 ; 0,3$ e $0,4 \mathrm{~L}^{-} \mathrm{ha}^{-1}$, utilizando-se pulverizador costal de CO2 com pressão de $40 \mathrm{lib} / \mathrm{pol}^{2}$, com barra dotada com um bico-leque 110:02 VS Teejet, com volume de aplicação utilizado de 300 L.ha $^{-1}$.

A coleta de folhas do algodoeiro para análise foliar seguiu as recomendações de SiLva e RAIJ (1996) e o método de análise recomendado por BAtAglia et al. (1983). Avaliou-se a altura da planta por ocasião da colheita, pela mensuração do seu comprimento da base até o ápice da planta, considerando-se dez plantas tomadas ao acaso por parcela.

A produtividade foi obtida através da colheita das duas linhas centrais de cada parcela. Foram colhidas amostras de 20 capulhos do terço médio das plantas, as quais foram beneficiadas, ou seja, a fibra separada das sementes. Assim, permitiu-se avaliar a massa de capulho (MC), massa de sementes (MS) e a porcentagem de fibra $(\% \mathrm{~F})$. Os dados foram submetidos à análise da variância, com análise conjunta dos experimentos (GOMEs, 2000), sendo as médias comparadas pelo teste de Tukey, ao nível de $5 \%$ de probabilidade.

\section{RESULTADOS E DISCUSSÃO}

O quadro 1 indica os valores de significância estatística $(\mathrm{P}>\mathrm{F})$ obtidos para as diferentes variáveis avaliadas. Verificou-se que houve efeito significativo de doses de $\mathrm{N}$ sobre a porcentagem de fibra, o teor foliar de $\mathrm{N}$ e sobre a produtividade de algodão.

Em relação ao uso do regulador vegetal, observou-se efeito significativo sobre a massa de capulhos e altura de plantas, enquanto a variável densidade de plantas afetou a massa de capulhos e a porcentagem de fibra. Não foram obtidas interações duplas ou triplas significativas. 
Quadro 1. Valores da Prob. > F obtidos na análise de variância conjunta para as características avaliadas nos experimentos realizados em diferentes localidades e anos agrícolas

\begin{tabular}{|c|c|c|c|c|c|c|}
\hline Causas da Variação & $\mathrm{MC}^{(1)}$ & $\mathrm{MS}^{(2)}$ & $\% \mathrm{~F}^{(3)}$ & Altura $^{(4)}$ & $\mathrm{TN}^{(5)}$ & $\begin{array}{l}\text { Produtivi- } \\
\text { dade }^{(6)}\end{array}$ \\
\hline Dose de $N(N)$ & 0,9600 & 0,6755 & $0,0199 * *$ & 0,0649 & $0,0023^{* *}$ & $0,0245^{* *}$ \\
\hline Regulador (R) & $0,0001^{* *}$ & 0,0740 & 0,7710 & $0,0001^{* *}$ & 0,1859 & 0,0709 \\
\hline Densidade (D) & $0,0003^{* *}$ & 0,5993 & $0,0007^{* *}$ & 0,1531 & 0,5325 & 0,0549 \\
\hline$N * R$ & 0,7709 & 0,6197 & 0,9778 & 0,8650 & 0,0767 & 0,9311 \\
\hline$N * D$ & 0,5134 & 0,5226 & 0,1754 & 0,6545 & 0,6201 & 0,7082 \\
\hline $\mathrm{R} * \mathrm{D}$ & 0,0736 & 0,2929 & 0,8953 & 0,7623 & 0,5503 & 0,6147 \\
\hline$N * R * D$ & 0,1820 & 0,3498 & 0,0989 & 0,5245 & 0,8911 & 0,9658 \\
\hline C.V. & 7,56 & 14,76 & 6,65 & 8,47 & 7,18 & 13,05 \\
\hline Média Geral & 133,34 & 12,09 & 42,91 & 97,38 & 43,06 & 2,90 \\
\hline
\end{tabular}

$\left({ }^{1}\right)$ Massa de 20 capulhos. $\left({ }^{2}\right)$ Massa de 100 sementes. $\left({ }^{3}\right)$ Porcentagem de fibra. $\left({ }^{4}\right)$ Altura de plantas. $\left({ }^{5}\right)$ Teor foliar de N. $\left({ }^{6}\right)$ Produtividade de algodão em caroço.

No quadro 2, pode-se verificar que a massa de capulhos apresentou uma tendência de aumentar com o parcelamento da aplicação de regulador $(+2,8 \%)$, o que pode estar relacionado ao fato do regulador, desde os estádios iniciais de desenvolvimento, reduzir o desenvolvimento vegetativo e promover melhor formação de maçãs. Tais resultados são concordantes com os obtidos por FERRAz et al. (1977), que verificaram maiores valores de massa de capulhos com aplicação de regulador vegetal. Por outro lado, houve decréscimo significativo nos valores dessa característica quando se aumentou a densidade de plantas por metro de 6 para $10(-2,3 \%)$ e para $14(-3,2 \%)$, fato que pode ter ocorrido devido à competição intraespecífica, provocando redução da massa de capulhos, o que concorda com os resultados obtidos por Cia et al.
(2001). Para as doses de nitrogênio, não se verificou efeito significativo, o que concorda com relato de JACKSON e GERIK (1990), que não observaram correlação alta entre a fertilização nitrogenada e a massa de capulhos.

Embora tenha ocorrido aumento na massa de sementes devido à utilização da aplicação parcelada de regulador $(+5,3 \%)$, tal diferença não foi significativa (quadro 3), concordando com Ferraz et al. (1977). Fato semelhante ocorreu com efeitos da adubação nitrogenada e com densidade de plantas, o que pode ser explicado devido à estabilidade da massa de 100 sementes, embora se tenha constatado uma redução na característica com o aumento do estande (CIA et al., 2001).

Quadro 2. Valores médios da massa de 20 capulhos (g) obtido para localidades no Estado de São Paulo em diferentes anos agrícolas

\begin{tabular}{|c|c|c|c|c|c|c|}
\hline \multirow{2}{*}{$\begin{array}{l}\text { Aplicação de } \\
\text { Regulador }\end{array}$} & \multirow{2}{*}{$\begin{array}{l}\text { Dose } \\
\text { de N }\end{array}$} & \multicolumn{3}{|c|}{ Densidade } & \multirow{2}{*}{$\begin{array}{l}\text { Médias de } \\
\text { Dose de N }\end{array}$} & \multirow{2}{*}{$\begin{array}{l}\text { Média de } \\
\text { Regulador }\end{array}$} \\
\hline & & 6 & 10 & 14 & & \\
\hline \multirow[t]{2}{*}{ Única } & 40 & 131,16 & 132,99 & 129,90 & 133,32 & 131,49 \\
\hline & 60 & 134,84 & 131,37 & 128,69 & $(40)$ & única \\
\hline \multirow[t]{2}{*}{ Parcelada } & 40 & 139,06 & 132,67 & 134,11 & 133,36 & 135,18 \\
\hline & 60 & 138,09 & 133,82 & 133,33 & 60 & parcelada \\
\hline Média de densidade & & $135,79 a$ & $132,71 b$ & $131,51 b$ & & \\
\hline
\end{tabular}

Médias seguidas por letras distintas diferem entre si ao nível de 5\% pelo teste de Tukey . 
Quadro 3. Valores médios da massa de 100 sementes (g) obtidos para localidades no Estado de São Paulo em diferentes anos agrícolas

\begin{tabular}{|c|c|c|c|c|c|c|}
\hline \multirow{2}{*}{$\begin{array}{l}\text { Aplicação de } \\
\text { Regulador }\end{array}$} & \multirow{2}{*}{$\begin{array}{l}\text { Dose } \\
\text { de N }\end{array}$} & \multicolumn{3}{|c|}{ Densidade } & \multirow{2}{*}{$\begin{array}{l}\text { Médias de } \\
\text { Dose de N }\end{array}$} & \multirow{2}{*}{$\begin{array}{l}\text { Média de } \\
\text { Regulador }\end{array}$} \\
\hline & & 6 & 10 & 14 & & \\
\hline \multirow[t]{2}{*}{ Única } & 40 & 11,69 & 11,83 & 11,77 & $11,92 \mathrm{a}$ & $11,78 \mathrm{a}$ \\
\hline & 60 & 11,97 & 11,74 & 11,68 & $(40)$ & única \\
\hline \multirow[t]{2}{*}{ Parcelada } & 40 & 12,10 & 11,97 & 12,14 & $12,26 a$ & $12,40 \mathrm{a}$ \\
\hline & 60 & 12,12 & 11,96 & 14,10 & $(60)$ & parcelada \\
\hline Média de densidade & & 11,97 & 11,88 & 12,42 & & \\
\hline
\end{tabular}

Médias seguidas por letras distintas diferem entre si ao nível de 5\% pelo teste de Tukey .

No quadro 4, verifica-se que o modo de aplicação do regulador vegetal não afetou significativamente a porcentagem de fibra. Por outro lado, a maior dose de $\mathrm{N}$ em cobertura propiciou um valor médio de porcentagem de fibra significativamente inferior àquele obtido com a dose de $40 \mathrm{~kg} \cdot \mathrm{ha}^{-1}$ de $N(-1,4 \%)$. Da mesma forma, verifica-se que maiores densidades (10 e 14 plantas por metro) conduziram a valores progressivamente menores de porcentagem de fibra, o que concorda com os resultados de WILKES e Corley (1968), Hawkins e Peacock (1970), Bridge et al. (1973), Ferraz et al. (1977), Belletini e Abrahão (1988) e CiA et al. (2001), que verificaram uma redução da porcentagem de fibra com o aumento da densidade de semeadura.

Os resultados evidenciam que a redução da massa de capulhos (Quadro 2), pelo aumento da densidade de semeadura, ocorre devido à diminuição da porcentagem de fibra, uma vez que a massa de sementes não é afetada por essa variável.

A altura das plantas (Quadro 5), não foi afetada pela dose de $\mathrm{N}$ em cobertura e pelas variações na densidade de plantas, o que discorda dos resultados obtidos por Belletini e ABRAhão (1988). No entanto, a aplicação parcelada de regulador reteve significativamente o crescimento do algodoeiro $(-11,4 \%)$.

A utilização gradativa do regulador de crescimento, aliando a dose ao estádio fenológico, exerceu efeito positivo na inibição de crescimento. A diferença de $12 \%$ em altura obtida com o uso de regulador é útil, principalmente quando se refere à necessidade de adequação para colheita mecanizada da cultura.

Os valores médios dos teores foliares de nitrogênio (Quadro 6), aumentaram com a aplicação da maior dose de $\mathrm{N}(+2,9 \%)$, e não foram significativamente modificados pelo sistema de aplicação de regulador e pela densidade de plantas. Tais resultados são concordantes com aqueles obtidos por OliveIRA e BALBINo (1995), em estudos realizados no Paraná, que verificaram valores de 39,2 e 38,4 g. $\mathrm{kg}^{-1}$ de $\mathrm{N}$, respectivamente, para doses de 25 e $75 \mathrm{~kg} \cdot \mathrm{ha}^{-1}$ de $\mathrm{N}$ aplicado. Os valores de $\mathrm{N}$ são considerados adequados de acordo com os relatos de TRANI et al. (1983), Malavolta et al. (1997).

Quadro 4. Valores médios da porcentagem de fibra obtidos para localidades no Estado de São Paulo em diferentes anos agrícolas

\begin{tabular}{|c|c|c|c|c|c|c|}
\hline \multirow{2}{*}{$\begin{array}{l}\text { Aplicação de } \\
\text { Regulador }\end{array}$} & \multirow{2}{*}{$\begin{array}{l}\text { Dose } \\
\text { de } N\end{array}$} & \multicolumn{3}{|c|}{ Densidade } & \multirow{2}{*}{$\begin{array}{l}\text { Médias de } \\
\text { Dose de N }\end{array}$} & \multirow{2}{*}{$\begin{array}{l}\text { Média de } \\
\text { Regulador }\end{array}$} \\
\hline & & 6 & 10 & 14 & & \\
\hline \multirow[t]{2}{*}{ Única } & 40 & 43,26 & 43,15 & 43,10 & $43,21 \mathrm{a}$ & $42,87 a$ \\
\hline & 60 & 43,91 & 42,00 & 41,81 & $(40)$ & única \\
\hline \multirow[t]{2}{*}{ Parcelada } & 40 & 44,05 & 42,72 & 42,98 & $42,61 b$ & $42,95 a$ \\
\hline & 60 & 43,27 & 42,88 & 41,78 & 60 & parcelada \\
\hline Média de densidade & & $43,62 \mathrm{a}$ & $42,68 b$ & $42,42 b$ & & \\
\hline
\end{tabular}

Médias seguidas por letras distintas diferem entre si ao nível de 5\% pelo teste de Tukey . 
Quadro 5. Valores médios da altura de plantas obtidos para localidades no Estado de São Paulo em diferentes anos agrícolas

\begin{tabular}{|c|c|c|c|c|c|c|}
\hline \multirow{2}{*}{$\begin{array}{l}\text { Aplicação de } \\
\text { Regulador }\end{array}$} & \multirow{2}{*}{$\begin{array}{l}\text { Dose } \\
\text { de N }\end{array}$} & \multicolumn{3}{|c|}{ Densidade } & \multirow{2}{*}{$\begin{array}{l}\text { Médias de } \\
\text { Dose de N }\end{array}$} & \multirow{2}{*}{$\begin{array}{l}\text { Média de } \\
\text { Regulador }\end{array}$} \\
\hline & & 6 & 10 & 14 & & \\
\hline \multirow[t]{2}{*}{ Única } & 40 & 103,79 & 103,22 & 101,03 & 96,72 & $103,28 \mathrm{a}$ \\
\hline & 60 & 104,44 & 102,94 & 104,24 & $(40)$ & única \\
\hline \multirow[t]{2}{*}{ Parcelada } & 40 & 91,25 & 91,32 & 89,71 & $42,61 b$ & $42,95 a$ \\
\hline & 60 & 93,05 & 92,67 & 90,84 & 60 & parcelada \\
\hline Média de densidade & & 98,13 & 97,54 & 96,46 & & \\
\hline
\end{tabular}

Médias seguidas por letras distintas diferem entre si ao nível de 5\% pelo teste de Tukey .

Quadro 6. Valores médios do teor foliar de nitrogênio (g. $\mathrm{kg}^{-1}$ ) obtido para localidades no Estado de São Paulo em diferentes anos agrícolas

\begin{tabular}{|c|c|c|c|c|c|c|}
\hline \multirow{2}{*}{$\begin{array}{l}\text { Aplicação de } \\
\text { Regulador }\end{array}$} & \multirow{2}{*}{$\begin{array}{l}\text { Dose } \\
\text { de N }\end{array}$} & \multicolumn{3}{|c|}{ Densidade } & \multirow{2}{*}{$\begin{array}{l}\text { Médias de } \\
\text { Dose de N }\end{array}$} & \multirow{2}{*}{$\begin{array}{l}\text { Média de } \\
\text { Regulador }\end{array}$} \\
\hline & & 6 & 10 & 14 & & \\
\hline \multirow[t]{2}{*}{ Única } & 40 & 42,25 & 41,86 & 41,37 & $42,44 b$ & 42,80 \\
\hline & 60 & 44,48 & 43,41 & 43,45 & $(40)$ & única \\
\hline \multirow[t]{2}{*}{ Parcelada } & 40 & 43,12 & 43,14 & 42,90 & $43,69 a$ & 43,33 \\
\hline & 60 & 43,67 & 43,02 & 44,12 & 60 & parcelada \\
\hline Média de densidade & & 43,38 & 42,86 & 42,96 & & \\
\hline
\end{tabular}

Médias seguidas por letras distintas diferem entre si ao nível de 5\% pelo teste de Tukey .

Na produtividade de algodão em caroço contida no quadro 7, pode-se constatar que o sistema de aplicação do regulador vegetal não exerceu efeito significativo. Tal fato é importante, visto que se pode adequar melhor a arquitetura da planta, através da redução da sua altura (Quadro 5) com a aplicação parcelada do cloreto de mepiquat sem alterar a produtividade.

Da mesma forma, a densidade de semeadura não modificou significativamente os valores de produção, concordando com CiA et al. (2001), que estudaram densidades de plantas variando de 5,97 até
13,84 plantas por metro. Por outro lado, no estudo realizado por MoRESCO et al. (1999a), utilizando 6 a 12 plantas por metro quadrado, houve aumento da produtividade com menor número de plantas. Os diferentes resultados podem ser explicados pelos espaçamentos entre linhas empregados nos diferentes estudos. No presente trabalho, utilizou-se um espaçamento que variou de 0,9 até $1,0 \mathrm{~m}$ nas diferentes localidades, enquanto no trabalho desenvolvido por MoRESCO et al. (1999b) foram obtidas produtividades inferiores na densidade de 12 plantas por metro, devido ao espaçamento de $0,76 \mathrm{~m}$ entre linhas de semeadura.

Quadro 7. Valores médios da produtividade de algodão em caroço $\left(\mathrm{kg}_{\mathrm{g}} \mathrm{ha}^{-1}\right)$ obtido para localidades no Estado de São Paulo em diferentes anos agrícolas

\begin{tabular}{|c|c|c|c|c|c|c|}
\hline \multirow{2}{*}{$\begin{array}{l}\text { Aplicação de } \\
\text { Regulador }\end{array}$} & \multirow{2}{*}{$\begin{array}{l}\text { Dose } \\
\text { de N }\end{array}$} & \multicolumn{3}{|c|}{ Densidade } & \multirow{2}{*}{$\begin{array}{l}\text { Médias de } \\
\text { Dose de N }\end{array}$} & \multirow{2}{*}{$\begin{array}{l}\text { Média de } \\
\text { Regulador }\end{array}$} \\
\hline & & 6 & 10 & 14 & & \\
\hline \multirow[t]{2}{*}{ Única } & 40 & 2.850 & 2.890 & 2.780 & $2.870 \mathrm{~b}$ & 2.870 \\
\hline & 60 & 2.960 & 2.960 & 2.800 & $(40)$ & única \\
\hline \multirow[t]{2}{*}{ Parcelada } & 40 & 2.900 & 2.900 & 2.880 & $2940 a$ & 2930 \\
\hline & 60 & 3.010 & 2.960 & 2.930 & 60 & parcelada \\
\hline \multicolumn{2}{|c|}{ Média de densidade } & 2.930 & 2.920 & 2.850 & & \\
\hline
\end{tabular}

Médias seguidas por letras distintas diferem entre si ao nível de 5\% pelo teste de Tukey. 
Quadro 8. Valores médios da produtividade de fibra de algodão (kg.ha ${ }^{-1}$ ) obtidos para localidades no Estado de São Paulo em diferentes anos agrícolas

\begin{tabular}{|c|c|c|c|c|c|c|}
\hline \multirow{2}{*}{$\begin{array}{l}\text { Aplicação de } \\
\text { Regulador }\end{array}$} & \multirow{2}{*}{$\begin{array}{l}\text { Dose } \\
\text { de N }\end{array}$} & \multicolumn{3}{|c|}{ Densidade } & \multirow{2}{*}{$\begin{array}{l}\text { Médias de } \\
\text { Dose de N }\end{array}$} & \multirow{2}{*}{$\begin{array}{l}\text { Média de } \\
\text { Regulador }\end{array}$} \\
\hline & & 6 & 10 & 14 & & \\
\hline \multirow[t]{2}{*}{ Única } & 40 & $1.232,9$ & $1.247,0$ & $1.198,2$ & $1.240,1 \mathrm{a}$ & $1.230,4 \mathrm{a}$ \\
\hline & 60 & $1.299,7$ & $1.243,2$ & $1.170,7$ & $(40)$ & única \\
\hline \multirow[t]{2}{*}{ Parcelada } & 40 & $1.277,5$ & $1.238,9$ & $1.237,8$ & $1.252,7 \mathrm{a}$ & $1.258,4 \mathrm{a}$ \\
\hline & 60 & $1.302,4$ & $1.269,2$ & $1.224,2$ & $(60)$ & parcelada \\
\hline \multicolumn{2}{|c|}{ Média de densidade } & $1.278,1 \mathrm{a}$ & $1.246,3 \mathrm{a}$ & $1.209,0 \mathrm{a}$ & & \\
\hline
\end{tabular}

Médias seguidas por letras distintas diferem entre si ao nível de 5\% pelo teste de Tukey.

A dose de nitrogênio de $60 \mathrm{~kg} \cdot \mathrm{ha}^{-1}$ em cobertura propiciou significativo aumento de produtividade $(+4,6 \%)$ quando comparada à menor dose de N (40 $\left.\mathrm{kg} \cdot \mathrm{ha}^{-1}\right)$. Por outro lado, não houve efeito significativo de doses, densidade ou sistema de aplicação do regulador vegetal sobre a produtividade de fibra (Quadro 8).

\section{CONCLUSÃO}

1. A aplicação parcelada de regulador vegetal é mais eficiente que a aplicação única, em termos de limitação do crescimento do algodoeiro e propicia maior massa média de capulhos. A aplicação da maior dose de $\mathrm{N}$ proporciona aumentos nos valores de $\mathrm{N}$ foliar e na produtividade de algodão, enquanto ocasiona diminuição na porcentagem de fibra. O aumento da densidade de plantas de 6 para 10 e 14 plantas por metro promove uma redução nos valores médios de porcentagem de fibra e da massa de capulhos.

\section{REFERÊNCIAS BIBLIOGRÁFICAS}

BATAGLIA,O.C.; FURLANI, A.M.C.; TEIXEIRA, J.P.F.; FURLANI, P.R.; GALLO, J.R. Métodos de análise química de plantas. Campinas: Instituto Agronômico, 1983. 48p. (Boletim Técnico, 78)

BELLETTINI, S.; ABRAHÃO, J. Influência de espaçamentos e distribuições espaciais sobre o rendimento de plantas de algodão 'IAC-20'. In: REUNIÃO NACIONAL DO ALGODÃO, 5., Campina Grande, 1988. Resumos... Campina Grande: EMBRAPA, 1988. p.75.

BRIDGE, R.R.; MEREDITH JUNIOR, W.R.; CHISM, J.F. Influence of planting method and plant population on cotton. Agronomy Journal, Madison, v.65, p.104-109, 1973.
CARVALHO, L.H.; FURLANI JUNIOR, E. Sistema de produção do algodão mecanizado. In: SEMINÁRIO ESTADUAL COM A CULTURA DO ALGODÃO EM MATO GROSSO, 1996, Cuiabá. Anais... Cuiabá: EMPAER-MT, 1996. p.105-113.

CIA, E.; FURLANIJUNIOR, E.; SABINO, N.P.;FUZATTO, M.G.; FERRAZ, C.A.M.; CARVALHO, L.H.; BORTOLETTO, N.; SABINO, J.C.; CAMPANA, M.P. Eliminação do desbaste na cultura do algodoeiro. Pesquisa Agropecuária Brasileira, Brasília, v.36, n.10, p.1239-1244, 2001.

FERRAZ, C.A.M.; CIA, E.; SABINO, N.P.; GROSSI, J.M.M. VEIGA, A.A.; YOSHIDA, H. Efeito da densidade de plantio e da aplicação de CCC em algodoeiro. Bragantia, Campinas, v.36, p.239-251, 1977.

GOMES, F.P. Curso de Estatística Experimental. Piracicaba: ESALQ/Universidade de São Paulo, 2000. 477 p.

HAWKINS, B.S.; PEACOCK, H.A. Yield response of upland cotton to several spacing arrangements. Agronomy Journal, Madison, v.62, p.578-580, 1970.

JACKSON, B.S., GERIK, T.J. Boll shedding and boll load in nitrogen: stressed cotton. Agronomy Journal, Madison, v.82, p.483-488, 1990 .

MALAVOLTA, E.; VITTI, G.C.; OLIVEIRA, S.A. Avaliação do estado nutricional das plantas. Piracicaba: POTAFÓS, 1997.319p.

MORESCO, E.R.; FARIAS, F.J.C.; SOUZA, M.; AGUIAR, P.H. MARQUES, M.F.; TAKEDA, A.C. Influência da densidade e do espaçamento na produtividade do algodoeiro herbáceo. In: CONGRESSO BRASILEIRO DE ALGODÃO, 2., 1999, Ribeirão Preto. Anais... Campina Grande: EMBRAPA-CNPA, 1999a. p. 629-631.

MORESCO, E.R.; FARIAS, F.J.C.;SOUZA, M.; MARQUES, M.F.; TAKEDA, C. Influência da densidade e do espaçamento na produtividade do algodoeiro herbáceo I. In: CONGRESSO BRASILEIRO DE ALGODÃO, 2., 1999, Ribeirão Preto. Anais... Campina Grande: EMBRAPA-CNPA, 1999b. p.632-633. 
OLIVEIRA, E.F.; BALBINO, L.C. Efeitos de fontes e doses de Nitrogênio aplicados em cobertura nas culturas de trigo, milho e algodão. Cascavel: OCEPAR, 1995. p. 7-39. (Resultados de Pesquisa, 1/95)

SILVA, N.M., RAIJ, B. van. Fibrosas. In: RAIJ, B. van.; CANTARELLA, H.; QUAGGIO, J.A.; FURLANI, A.M.C. (Eds.). Recomendações de adubação e calagem para o Estado de São Paulo. Campinas: Instituto Agronômico \& Fundação IAC, 1996. p.107111. (Boletim Técnico, 100)
TRANI, P.E., HIROCE, H., BATAGLIA, O.C. Análise foliar: amostragem e interpretação. Campinas: Fundação Cargill, 1983. 18p.

WILKES, L.H., CORLEY, T.E. Planting and cultivation. In: ELLIOT, F.E., HOOVER, M., PORTER JUNIOR, W.K. (Eds.). Advances in production and utilization of qualit 'cotton': principles and pratices. Ames: Iowa State University Press, 1968. p. 115-117. 